\title{
Control and Communication Techniques for the Smart Grid: An Energy Efficiency Perspective
}

\author{
Yee Wei Law ${ }^{*}$ Hemanshu R. Pota** Jiong Jin*** \\ Zhihong Man ${ }^{* * *}$ Marimuthu Palaniswami* \\ * Department of Electrical and Electronic Engineering, The University \\ of Melbourne, Parkville, VIC 3010, Australia (e-mail: \\ $\{$ ywlaw,palani\}@unimelb.edu.au) \\ ** School of Information Technology and Electrical Engineering, The \\ University of New South Wales, Canberra ACT 2600, Australia \\ (e-mail: h.pota@adfa.edu.au) \\ *** Faculty of Engineering and Industrial Sciences, Swinburne \\ University of Technology, Hawthorn, VIC 3122, Australia (e-mail: \\ $\{$ jiongjin,zman\}@swin.edu.au)
}

\begin{abstract}
Energy is a major driver of developments in human society. Naturally, the energy efficiency of power grids qualifies as an important topic. Half a century old, the world's power grids are due for a major overhaul. The envisioned energy-efficient, environment-friendly, distributed-generation-friendly, demand-response-friendly, self-healing power grid is called the smart grid. This article is intended as a primer on the control and communication techniques that form the basis of several smart grid subsystems, namely distributed generation, wide-area monitoring, distribution automation, and substation automation. The theme of this article is to show how these techniques contribute to the efficiency of the generation, transmission, and distribution segments of a grid. Potential research challenges and opportunities are indicated. To complement our technical discussion, noteworthy smart grid developments in Australia (one of the world's top investors in smart grids) are highlighted.
\end{abstract}

Keywords: Smart grid, energy efficiency, distributed generation, V2G, microgrid, wide-area monitoring, distribution automation, substation automation

\section{INTRODUCTION}

In Australia, $75 \%$ of the energy consumed still comes from coal-based power stations, making Australia one of the highest greenhouse gas emitters per capita. Furthermore, Australia's technical energy-efficiency improvement between 1990 and 2004 was only about a third of the average amongst OECD countries. The below-average performance has motivated Australia to set a National Energy Efficiency Target to achieve world-class saving by 2015. It is estimated that if power grids were $5 \%$ more efficient, the energy savings would equate to permanently eliminating the fuel and greenhouse gas emissions from 53 million cars (U.S. Department of Energy, 2008).

Since line loss is proportional to the current squared, a grid is more energy-efficient with lower demand, and therefore load reduction is an obvious way of improving a grid's energy efficiency but this approach depends mostly on market forces. Flattening the demand curve is another way of making a grid more energy-efficient. To understand this, consider a load that draws a current of $2 i$ for half of the day, but no current for the rest of the day, and thereby incurring a line loss that is proportional to $(2 i)^{2} \times$ $\frac{1}{2}$ day. Consider another load that draws a current of $i$ throughout the day, and thereby incurring a line loss that is proportional to $i^{2} \times 1$ day. The latter load which represents a flat demand incurs half as much line loss. Therefore, a flatter demand curve is better for energy efficiency, besides infrastructure utilization.

Based on the analysis above, the smart grid is meant to provide various means of suppressing power wastage and fluctuations. IEEE Std 2030-2001 defines the smart grid as "the integration of power, communications, and information technologies for an improved electric power infrastructure serving loads while providing for an ongoing evolution of end-use applications." By exploiting multidisciplinary techniques to admit renewable energy and improve the energy efficiency of power grids, and thereby reducing greenhouse gas emission, smart grids will address some of the most pressing needs of our time.

A general overview of the smart grid has been presented by Massoud Amin and Wollenberg (2005); Ipakchi and Albuyeh (2009); Farhangi (2010); Andersson et al. (2011). Discipline-specific surveys include the following: Wang et al. (2011) and Gao et al. (2012) target communication architectures and technologies in smart grids; Tan et al. (2011) target machine-to-machine (M2M) applications in smart grids; and Venayagamoorthy (2011) targets applications of computational intelligence to smart grids. 
This review focuses on the energy efficiency of the smart grid, and covers multidisciplinary techniques in relation to energy efficiency. This review is written with the goal of keeping researchers outside the power system discipline abreast of the latest developments in smart grids amidst the recent flurry of research activities. This review is also written so that power system researchers can learn about technologies that are not usually associated with power systems but play a role in shaping smart grids. Furthermore, this review complements Law et al.'s (2012) review on demand-side techniques.

In terms of organization, Sections 2, 3 and 4 discuss generation-side, transmission-side and distribution-side techniques respectively. Section 5 presents research challenges and opportunities. Section 6 concludes. A list of acronyms is provided in Table 1.

\section{GENERATION-SIDE TECHNIQUES}

A generation process is energy-efficient if it incurs little energy loss in converting a non-electrical energy source into electrical energy. Fossil fuels are exhaustible regardless of how efficient the process is, thus the long-term solution is renewable resources that are available in large stable quantity and that allow efficient generation of electricity. New market mechanisms (e.g., carbon markets), new funding mechanisms (e.g., government subsidies), environmental concerns and improving technologies are making distributed generation (DG) of renewable energy an appealing proposition. For example, the Australian parliament passed a nationwide portfolio standard in 2009, requiring the country to produce 20 per cent of its electricity from renewable resources by 2020 .

DG plants are also called distributed energy resources (DERs). The International Council on Large Electric Systems (CIGRE) Working Group 37-23 defines DERs as "non-predictable, non-dispatchable small generation units (nominal power below $50 \mathrm{MW}$ ), connected to distribution power systems." However, Ackermann et al.'s (2001) simpler definition of DG as "an electric power source connected directly to the distribution network or on the customer site of the meter" is apt enough. Most DERs are connected at the distribution level of a grid to minimize loss. We first discuss the issues of connecting a single DER to a grid, then the issues of connecting multiple DERs to a grid in terms of the penetration of DG.

\subsection{DER-grid interconnection}

Power grids were not originally designed to accommodate active generation and storage at the distribution level, so connecting DERs to a grid requires new technologies. In general, a DER-grid interconnection consists of an inputside converter on the DG side, and a grid-side converter on the grid side, controlled by an input-side controller and a grid-side controller respectively (see Fig. 1) (Timbus et al., 2009). The function of the input-side controller is to extract maximum power from the energy source, and to protect the DG in case of grid failures. The gridside controller handles power quality and voltage variation issues.

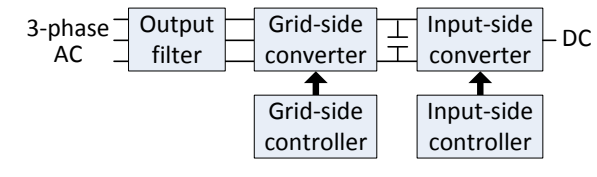

Fig. 1. A typical DER-grid connection.

Power quality issues: Transmission system operators (TSOs) usually impose stringent demands on connecting DERs. For example, for wind DG, the maximum limit for total harmonic distortion of the output current is $5 \%$ according to the IEEE Std 1547-2003. Experimental results show that a proportional-integral controller offers sufficient performance for filtering unwanted harmonics (Timbus et al., 2009).

Voltage variation issues: TSOs also require DERs to satisfy the ride-through time requirements for voltage variations, e.g., a wind DER is required to ride through a $25 \%$ voltage dip for $0.1 \mathrm{~s}$. Experimental results show that a deadbeat controller is sufficiently robust in case of a grid fault (Timbus et al., 2009). A deadbeat controller is a discrete-time controller giving zero steady-state error, minimum rise time and settling time, and less than $2 \%$ of overshoot or undershoot (Dorf and Bishop, 2008). The simplest transfer function for a deadbeat controller is:

$$
G(z)=z^{-1}
$$

i.e., delaying the input by just one sampling interval (it is infeasible to delay by less than one sampling interval). In fact, (1) can be used to relate grid-side converter voltage to grid voltage (Timbus et al., 2009, (7) and (8)). For mechanical systems, deadbeat controllers may cause excessive wear and tear due to sudden changes in the input/output, but for electrical systems, it is acceptable and it has been used since the 1980 s to control pulse widthmodulated inverters (Kawamura et al., 1988).

\subsection{Penetration of $D G$}

In May 2010, a storm caused the wind farms in Oregon to generate "almost two nuclear plants worth" of energy. The unprecedented output was a blessing had the grid been able to absorb it (Sickinger, 2010). Integrating DG with a main grid presents a number of challenges, due to two fundamental reasons: (i) power grids were originally designed to be sole exporters of energy; (ii) DG is inherently unstable in terms of output characteristics (e.g., wind or solar generation varies with meteorological conditions). In the literature, integration of DG in a grid is called penetration of DG.

Voltage fluctuation is one of the main hurdles to the penetration of DG. Penetration level is defined as the ratio of the amount of DG energy injected into the grid to the feeder capacity (Quezada et al., 2006). In general, the higher the penetration level, the worse the fluctuation becomes. There is hence a need to determine the maximum allowable penetration level of DG that current distribution networks can support. So far, it is only known that as a general rule, problems start appearing when the total connected DG capacity approaches $15 \%$ of the feeder capacity, but even this rule of thumb does not apply to special cases, such as when the DERs are clustered near a substation (Santoso et al., 2002). Quezada et al.'s 
Table 1. Frequently used acronyms in alphabetical order.

\begin{tabular}{|ll|}
\hline DA & Distribution automation \\
DER & Distributed energy resource \\
DG & Distributed/dispersed generation \\
EMS & Energy management system \\
EPRI & Electric Power Research Institute \\
EV & Electric vehicle \\
\hline
\end{tabular}

(2006) detailed analysis shows that energy loss presents a U-shaped trajectory against penetration level, i.e., it decreases with penetration level to a point (usually $<10 \%$ ) where it starts increasing with penetration level.

In order to determine the maximum penetration level, it is necessary to control voltage fluctuations. According to IEEE Std 1547-2003, DERs should not actively regulate distribution system voltages as it may destabilize the onload tap changers of some of the distribution transformers, but coordinated voltage regulation between utilities and DERs is allowed.

The starting point of a voltage control scheme is typically optimal power flow. A power flow problem, normally formulated as a nonlinear system of equations, is a problem of calculating the voltage and phase at each bus of a power system under balanced three-phase steady-state conditions. An optimal power flow problem is an optimization problem with a cost function that typically models operation cost, and constraints that are determined by the physical and operational characteristics (e.g., voltage and current ratings) of the power system.

Vovos et al.'s (2007) distributed voltage control scheme is based on optimal power flow. By writing the cost function as a quadratic function of DG capacity with negative coefficients, and relaxing the power factor constraint of DERs (as opposed to fixing the power factor in the socalled power factor control mode, as distribution network operators typically require), they show that distributed voltage control can increase penetration level by $72 \%$ compared to if rigid power factor control is used. By writing the power flow equations in a particular form, Ayres et al. (2010) show that the maximum amount of power that DERs can inject into each system bus without violating voltage limit can be efficiently calculated.

Besides controlling voltage, the location and size of each DER are also important design factors. When a DER is sized to closely match the local load and is located near the load, it can provide a significant reduction in line losses. The concept of microgrids (more on this later) was born of this preference for locality. Moreover, the more dispersed DERs are along network feeders, the higher the loss reduction becomes. If a DER is located far from a substation and delivers power toward the substation or even back to the transmission network through the substation transformer, losses can increase in the distribution system (Quezada et al., 2006). Lee and Park (2009) suggest installing a DER at the location of a capacitor bank. If the DER has proper reactive power control, it has the same effect on the system as the capacitor bank (see Section 4 about the application of capacitor banks).

In terms of size, the ideal condition for minimum loss is when the total amount of power generated equals the

\begin{tabular}{ll|} 
FTTP & Fiber-to-the-pole \\
PDC & Phasor data concentrator \\
PMU & Phasor measurement unit \\
SA & Substation automation \\
V2G & Vehicle-to-grid \\
WAMS & Wide-area monitoring/measurement system \\
\hline
\end{tabular}

amount of power consumed. The problem is to find the size of each DER giving the minimum total power loss. Define $\boldsymbol{x}$ as the vector whose $i$ th element represents the size of the $i$ th DER, then the total power loss is a nonlinear function of $\boldsymbol{x}$, denoted by $f(\boldsymbol{x})$. The problem then becomes:

$$
\min f(\boldsymbol{x}) \text { s.t. }\left[\begin{array}{llll}
1 & 1 & \cdots & 1
\end{array}\right] \boldsymbol{x} \leq P_{\max }, \boldsymbol{x} \succeq 0 .
$$

In $(2), \succeq$ represents component-wise $\geq$, and $P_{\max }$ caps the total size of the DERs. Instead of solving the nonlinear optimization problem (2) directly, Lee and Park (2009) propose a heuristic method that generates a series of values of $f(\boldsymbol{x})$, and from the minimum of these values, uses Kalman filtering to estimate the corresponding $\boldsymbol{x}$.

Nevertheless, generation-to-load proximity is a doubleedged sword: while it reduces losses, small variations in the loads can excite voltage oscillations due to control interaction and reactive power mismatch. Recently, a new oscillation mode called the "critical voltage mode" has been discovered, which has a frequency between the frequency range of electromechanical oscillation and the frequency range of subsynchronous (torsional) oscillation (Roy et al., 2013a,b). This "critical voltage mode" must be damped to maintain secure and stable operations. In a distribution system, linearized generator models are not appropriate because load change is large in proportion to generation. Furthermore, the operating points are constantly changing. Thus, robust control is necessary. A linear quadratic Gaussian controller with norm-bounded uncertainty has been found to work well (Roy et al., 2013b).

Electric vehicles (EVs) with grid-to-vehicle $(\mathrm{G} 2 \mathrm{~V})$ and vehicle-to-grid (V2G) power-flow capability have the potential to absorb variations in renewable generation and lower maximum power transfer over transmission and distribution lines-this is discussed in Section 2.3. Despite these advances, it is generally recognized that for significant increase in penetration of DG, a major shift away from existing interconnection methods to microgrids (Lasseter, 2002) or comparable infrastructures is needed-this is discussed in Section 2.4.

\subsection{Vehicle-to-grid energy resources}

V2G refers to the provision of energy or ancillary services such as frequency regulation and voltage regulation from EVs to a electricity grid. Based on a V2G penetration level of 4 percent, the existing grid can be transformed into a smart grid exploiting the underutilized infrastructure consisting of EV batteries without needing high-cost investment in new transmission lines, transformers, and protection systems (Budischak et al., 2013). The research, innovation and tasks necessary for a successful adaptation of EVs are: (i) open-architecture hardware and software enabling V2G power flows; (ii) methods to obtain grid operating constraints using ISO ancillary supply pricing, demand response, EV user preference data, and power qual- 
ity requirements; and (iii) smart charging and discharging algorithms to satisfy the grid operating constraints.

An EV charger can be either unidirectional or bidirectional. A bidirectional charger can, not only charge but also, discharge an EV's battery to support V2G power flow. EVs can provide real power support to the grid in response to the grid operator's ancillary price signals or demand response events. Traditional unit commitment which considers only fuel cost, generator startup cost, generator shutdown cost in the cost function, can be extended to incorporate EVs as generating units. Saber and Venayagamoorthy (2010) propose incorporating carbon emission cost in the cost function. Similarly, optimal power flow has been extended to determine optimal G2V and V2G power flows (Acha et al., 2010). In all cases, the cost for incentivizing EV participation should be also considered, and this cost should include the cost of battery wear and other cost associated with V2G flows.

V2G power flow control can be accomplished within a unified control framework of DERs. An emerging trend is to represent/integrate EVs as DERs within IEC 61850, a series of standards for substation automation discussed in Section 4.2. This can be achieved by a mapping between ISO/IEC 15118, a widely adopted V2G communication interface standard, and IEC 61850-7-420 (Schmutzler et al., 2012).

Nevertheless, most EV chargers in existence are unidirectional, and will remain so in the foreseeable future. This limits V2G to providing only ancillary services. Although this can reduce V2G profits to less than $1 / 4$ of what bidirectional charging can offer (Tomić and Kempton, 2007), the benefits are still substational and are discussed in Section 4.

\subsection{Microgrids}

A microgrid is a cluster of interconnected DERs, storages and loads on a low-voltage distribution system, operating as a single controllable system that provides both power and heat to its local area. Microgrids significantly reduce feeder losses, and provide improved control of reactive power and voltage profile. However, the reduced energy losses and increased reliability come at the cost of increased control complexity.

One critical control objective is load sharing. The operation of a power system is based on the principle that the generators should share the loads in proportion to their ratings. Letting a small generator share a large proportion of the loads is inefficient, uneconomical, and poses the risk of tripping and causing cascading failures when the generator's capacity is exceeded. For conventional synchronous generators, load sharing is achieved through centralized automatic generation control, which distributes the generation task among the generators based on the system frequency. For DG units in a microgrid operating in the island mode (disconnected from main generators), load sharing becomes an issue because the units are not centrally controlled, and most of them are not synchronous generators. For small-scale and structurally simple distribution networks, load sharing can be achieved with low communication overhead between DG units (Marwali et al., 2004). For large-scale and complex distribution networks, communication-free load sharing schemes called droop control are more viable, but they permit a small control error. Existing droop controllers are also based on several assumptions, most notably the assumption that the DC voltage sources behind the inverters are ideal (Chung et al., 2010; He and Li, 2012). This assumption is valid for power sharing amongst parallel connected inverters due to limited interaction between sensor dynamics and controller dynamics, but it needs extension when the ideal voltage sources are replaced with renewable energy resources (Pota, 2013). Future research needs to consider generator dynamics, load dynamics, along with the sensor dynamics for a proper design of microgrid droop controllers.

As an extension to microgrids, LoCal grids are the power system analog of the Internet (He et al., 2008), and can be understood as an architecture for interconnecting microgrids. In the LoCal grid architecture, intelligent power switches operate as autonomous routers of "packetized energy", supplying or consuming energy depending on their neighbors as well as local supply, storage and demand. LoCal grids face certain challenges, the first of which being the establishment of a viable business model for energy import/export. Another challenge is coping with critical demand peaks. In this scenario, an intelligent power switch acts as an energy aggregator enforcing an energy rationing policy. The energy rationing problem - how to allocate power from a limited supply to satisfy relatively high demand - can be formulated as a queueing system problem (Lee et al., 2011), where the system parameters are

- the distribution of the interarrival time of customer requests;

- the distribution of the service time (duration);

- the maximum number of customers served at a time;

- the maximum length of the request queue;

- the size of the customer population.

Note that power $\times$ service time is equal to the amount of energy served to a customer in one service session. Lee et al. (2011) assume the existence of a pricing/incentive scheme such that the queueing model $M / M / c / K / m$ is applicable -in Kendall's notation, this means Markovian (exponentially distributed) interarrival times and service times, $c$ out of a total of $m$ customers can be served at a time, and a maximum of $K$ requests can be queued. By fixing the service time so that the queueing model $M / D / c$ is applicable (i.e., Markovian interarrival times, deterministic service times, $c$ customers are served at a time), Zhang and Baillieul (2013) show that the mean waiting time of a $M / D / c$ system can be made less than the mean waiting time of a $M / M / c$ system, provided the service time of the $M / D / c$ system is kept shorter than the mean service time of the $M / M / c$ system.

\section{TRANSMISSION-SIDE TECHNIQUES}

Typically about $8-10 \%$ of electrical energy appearing at generator terminals will be lost on its way to consumers in transmission and distribution networks. A transmission system has the important task of minimizing energy loss and maintaining system stability. Maintaining the health of the network by means of constant monitoring and control is therefore crucial. The starting point of monitoring 
is an energy management system (EMS). An EMS is the central nervous system of a transmission grid, giving utilities the ability to control generation; as well as aggregate, manage, and dispatch power at the transmission level. An EMS performs optimal power flow analysis, and recommends optimization actions. However, traditional SCADAbased EMS tends to give an incomplete view of the system steady state (Zima et al., 2005) - this inadequacy actually played a key role in the 2003 Northeastern Blackout and 2003 Italian Blackout.

A wide-area monitoring system (WAMS) is essentially a high-speed, hierarchical network of phasor measurement units (PMUs), whose sole objective is to report voltage and current phasor measurements (amplitude, frequency and phase). Given enough real-time phasors, the state of the grid (voltage and phase angle of each bus) can be tracked. A WAMS thus enhances the situational awareness of an existing SCADA-based EMS by adding real-time, wide-area monitoring, control and protection functionality (Martinez et al., 2005).

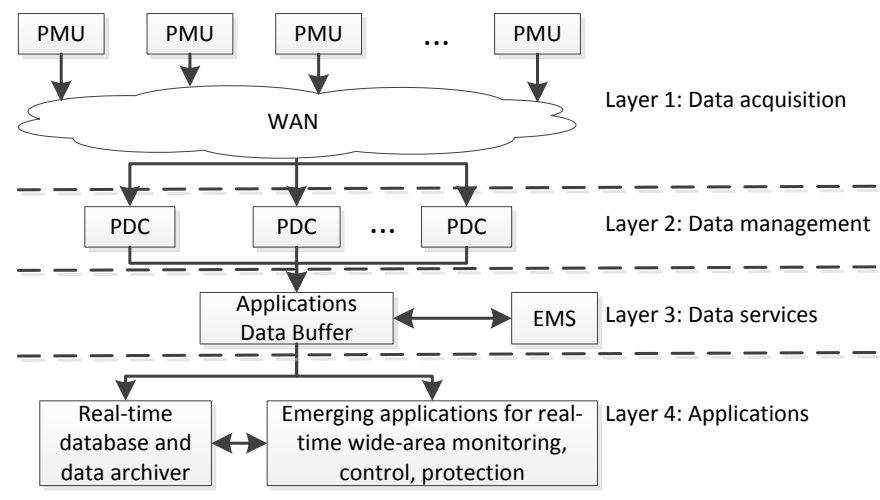

Fig. 2. The generic WAMS architecture.

A WAMS consists of four components: (i) synchronized phasor measurement units (synchronized PMUs or synchrophasors), (ii) phasor data concentrators (PDCs), (iii) a wide area network (WAN), and (iv) a real-time database and data archiver (Martinez et al., 2005). Figure 2 shows the four-layer generic architecture of a WAMS (Martinez et al., 2005). The PMUs in Layer 1 report voltage and current phasors at 10-25 frames per second for $50 \mathrm{~Hz}$ grids, 10-30 frames per second for $60 \mathrm{~Hz}$ grids; and utilize highly accurate common time sources such as the Global Positioning System (GPS) to time-tag the samples, enabling the correlation of phasor measurements across a wide grid area. The operation of a PMU is standardized in IEEE Std C37.118. The PMUs transmit phasor data to the PDCs in Layer 2 via a WAN. The PDCs correlate the time-tagged data, and forward the data to the data services in Layer 3. The data services monitor the data for loss, errors and synchronization, in addition to supplying the data in the required format to the applications in Layer 4. Layer 4 consists of a real-time database and data archiver, which is responsible for collecting and archiving data for postevent analysis and assessment. Layer 4 also consists of applications for monitoring, analysis, control and protection functions. These wide-area applications are categorized as oscillation control, voltage control, frequency control and line temperature monitoring functions. These applications are discussed next.
Wide-area oscillation control: Lack of damping torque at the generator rotors can cause oscillations in a grid. With real-time phasor measurements, it is now possible to detect poorly damped oscillations and take actions before they lead to system collapse. Traditionally, eigenvalue algorithms such as the QR algorithm, selective modal analysis, the AESOPS algorithm and the modified Arnoldi method are used to estimate the oscillation parameters (Huang et al., 2005). In the advent of the WAMS, it is feasible to implement more efficient algorithms such as Prony analysis (Hauer et al., 1990) and Kalman filteringthese algorithms are discussed below.

As a common starting point, we represent a signal as

$$
y[k]=y(k T)=\sum_{i=1}^{p} B_{i} e^{\lambda_{i} k T}=\sum_{i=1}^{p} B_{i} z_{i}^{k},
$$

where $k$ represents the $k$ th time step, $T$ is the sampling interval, $p$ is the number of oscillation modes, $\lambda_{i}$ is the eigenvalue of the $i$ th mode, and $z_{i} \triangleq e^{\lambda_{i} T}$. The number of modes $p$ can be determined by model selection, e.g., based on the Akaike Information Criterion. Based on the theory of linear differential equations, $z_{1}, \ldots, z_{p}$ are necessarily the roots of some $p$-th order characteristic equation with coefficients $a_{1}, \ldots, a_{p}$ :

$$
z^{p}-a_{1} z^{p-1} \cdots-a_{p}=0 .
$$

To use Prony analysis, we first establish that

$$
\left(\begin{array}{c}
y[0] \\
y[1] \\
\vdots \\
y[N]
\end{array}\right)=\left(\begin{array}{cccc}
1 & 1 & \cdots & 1 \\
z_{1} & z_{2} & \cdots & z_{p} \\
\vdots & \vdots & \ddots & \vdots \\
z_{1}^{N} & z_{2}^{N} & \cdots & z_{p}^{N}
\end{array}\right)\left(\begin{array}{c}
B_{1} \\
B_{2} \\
\vdots \\
B_{p}
\end{array}\right)
$$

Based on (4) and (3), Hauer et al. (1990) show that

$$
\left(\begin{array}{c}
y[p] \\
y[p+1] \\
\vdots \\
y[N-1]
\end{array}\right)=\left(\begin{array}{ccc}
y[p-1] & \cdots & y[0] \\
y[p] & \cdots & y[1] \\
\vdots & \ddots & \vdots \\
y[N-2] & \cdots & y[N-p-1]
\end{array}\right)\left(\begin{array}{c}
a_{1} \\
a_{2} \\
\vdots \\
a_{p}
\end{array}\right) \text {. }
$$

Provided $N \geq 2 p$ (Nyquist criterion), we can solve (5) for $a_{1}, \ldots, a_{p}$ using the method of least squares, and subsequently the roots $z_{1}, \ldots, z_{p}$ of $(3)$, as well as the eigenvalues $\lambda_{1}, \ldots, \lambda_{p}$. Finally, substituting $z_{1}, \ldots, z_{p}$ into (4) and solving (4) gives us $B_{1}, \ldots, B_{p}$.

To use Kalman filtering, we represent the a priori estimate $\hat{y}[k \mid k-1]$ as an autoregressive (AR) process of a finite order, $p$ :

$$
\hat{y}[k \mid k-1]=\sum_{i=1}^{p} a_{i} y[k-i] .
$$

This linear prediction model is general enough because every finite-order AR process can be expressed as an infinite-order moving average process. Observe that (6) is consistent with (5) if every $y[k]$ is replaced with $\hat{y}[k \mid k-1]$ on the left-hand side of (5). At this point, Korba (2007) applies the information form of the Kalman filter to estimate $a_{1}, \ldots, a_{p}$ by solving $\min _{a_{1} \ldots, a_{p}} \sum_{k=1}^{N}(\hat{y}[k \mid k-1]-y[k])^{2}$ iteratively. Korba (2007) proposes converting (3) into the continuous-time domain by Tustin approximation. Then, substituting $a_{1}, \ldots, a_{p}$ into the converted characteristic equation provides the eigenvalues $\lambda_{1}, \ldots, \lambda_{p}$ directly. 
Both Prony analysis and Kalman filtering give us the eigenvalues $\lambda_{1}, \ldots, \lambda_{p}$. From the eigenvalues, the $i$ th absolute damping factor $\sigma_{i}$ and oscillation frequency $f_{i}$ can then be obtained as $\sigma_{i}=\mathfrak{R e}\left(\lambda_{i}\right)$ and $f_{i}=\mathfrak{I m}\left(\lambda_{i}\right) /(2 \pi)$ respectively. Although Kalman filtering is more efficient than Prony analysis, the former can detect the most dominant oscillating mode accurately only if the damping factor is small, e.g., if the mode is unstable or nearly unstable (Peng and Nair, 2009a). As a batch processing algorithm, Prony analysis does not detect oscillation as quickly. As an improvement, the sampling interval $T$ can be adjusted adaptively (Peng and Nair, 2009b).

Wide-area voltage control: Grid voltage changes with load. Traditional undervoltage load shedding works by disconnecting load when the voltage falls below a preset threshold. Pre-setting threshold means relays cannot adapt to changing operating conditions (Larsson et al., 2002). Real-time phasor measurements make voltage instability assessment more efficient.

Wide-area frequency control: Mean grid frequency may change due to events causing large generation deficits such as generator outages (Karlsson et al., 2004). Traditional underfrequency load shedding operates offline, can be slow, and may lead to overfrequency due to excessive load shedding. Real-time phasor measurements make frequency instability assessment more efficient (Zima et al., 2005; Larsson et al., 2002).

Wide-area line temperature monitoring: The primary limitation on power flow is thermal. Since line temperature changes with transferred power, it can be estimated from phasor measurements and the line's material properties. Without line temperature monitoring, power restrictions based on temperature are pre-set at conservative limits considering worst case scenarios such as high ambient temperature, no wind, etc. With real-time monitoring of temperature, it is possible for power limits to be adjusted to allow maximum power flow (Zima et al., 2005). However, estimating line temperature from phasor measurements alone is not only inaccurate without taking ambient temperature into account, but also costly due to the high cost of PMUs. Increasing reliability requirements, increasing cost of scheduled maintenance, and increasing need for maximizing utility are driving the search for lowcost and scalable solutions for monitoring of not only the temperature, but also other parameters such as leakage current, lightning current, strain, vibration, and tilt of transmission lines. In other words, a WAMS needs to be complemented with an additional network of sensors for monitoring these parameters, and a few potential solutions are discussed below.

Line-traversing robots and unmanned aerial vehicles (i.e., drones) are among the most interesting proposals (Elizondo et al., 2010). However, most line-traversing robots are not only expensive, but are also unable to cross deadend structures and jumper cables. Manned helicopters are being replaced by drones ( $\mathrm{Li}$ et al., 2010), due to their low cost, but drones are encumbered with legal issues. For example, in Australia, flight periods, altitudes and distances must be approved by the Civil Aviation Safety Authority. Also, most drones are currently manually operated.
A more viable alternative is to deploy sensor networks on transmission lines and pylons (EPRI, 2009; Leon et al., 2007). Camera sensors on pylons can capture images of adjacent transmission lines to detect scratches, corrosion, changes in cable diameter, sagging, etc. Sensors on a transmission line naturally form a linear topology, but in this topology, packet latency is proportional to the maximum hop count of a sensor network. Therefore, Hung et al. (2010) propose equipping every node with multiple radios, namely ZigBee and GSM/GPRS/UMTS. Nodes close to a base station send their packets to the base station hop by hop using ZigBee. Selected nodes far from the base station collect packets from their neighborhood and "fast-track" its own and collected packets to the base station using cellular telephony at the expense of energy and cellular charges.

\subsection{Communication technologies for the WAMS}

PMUs spread over a large area are connected to PDCs via a WAN. To support the real-time monitoring and control functions just discussed, good performance of the WAN is crucial as these functions have strict time constraints (Martinez et al., 2005). The WAN can either be dedicated or shared.

Dedicated networks: PMUs in this type of network communicate with PDCs through the dedicated channels of a fiber-optic backbone connected to the substation LAN router. This configuration enjoys high capacity, low latency and low congestion, but at a high installation cost.

Shared networks: WANs based on TCP/IP implemented over shared network can also be used. The physical medium of these networks can be fiber-optic cables, copper lines or even wireless links. Cheaper and flexible software and hardware are available for TCP/IP-based networks. However, traffic from PMUs in a shared network has to compete for bandwidth with other lower-priority traffic such as VoIP, and yet TCP has no notion of quality of service. Moreover, standard TCP does not handle congestion well. Some research has been done to overcome these drawbacks of TCP for the WAMS. For example, Astrolabe (Birman et al., 2005) is a communication architecture that uses gossip-based routing (where a node rebroadcasts to forward a packet at a certain probability) for robustness. GridStat (Hauser et al., 2005) is a middleware framework that focuses on quality of service. The main advantage of shared networks is low cost.

As PDCs delay forwarding data until data from all PMUs is received, the PMU with the longest delay could become a bottleneck in the system. Furthermore, the installation of a PMU costs tens of thousands of dollars (De La Ree et al., 2010), the optimal placement of PMUs and PDCs is thus an important consideration. For a system with $N$ buses and $L$ branches, the optimal PMU placement problem can be formulated as an integer program:

$$
\min \mathbf{c}^{T} \boldsymbol{x} \text { s.t. } \boldsymbol{T} \boldsymbol{x} \succeq\left[\begin{array}{llll}
1 & 1 & \cdots & 1
\end{array}\right]^{T},
$$

where

c is a vector whose $i$ th element is the installation cost of a PMU on branch $i$;

$\boldsymbol{x}$ is a vector whose $i$ th element is 1 if a PMU is placed on branch $i$, and 0 if otherwise; 
$\boldsymbol{T}$ is a matrix whose $(i, j)$ th element is 1 if bus $i$ is connected to branch $j$, or 0 if otherwise;

$\succeq$ denotes component-wise $\geq$.

The inequality constraint in (7) is due to the requirement that for every bus, at least one of its branches has a PMU.

\section{DISTRIBUTION-SIDE TECHNIQUES}

Major causes of energy loss in the distribution network include (i) inductive reactive contributed by cables and transformers to the network, and (ii) fluctuation of loading. We first discuss power factor correction (PFC) as a solution for (i), and then integrated volt/VAr control (IVVC) and V2G ancillary services as solutions for (ii). Subsequently, we discuss distributed automation (DA) and substation automation (SA), which provide automation support for PFC, IVVC and V2G ancillary service.

Power factor correction (PFC): Inductive reactance in the network causes low power factors that result in preventable losses. The higher the inductive reactance, the greater the phase angle (the lower the power factor) and hence loss. The objective of PFC is to improve power factor by adding (series and shunt, but mostly shunt) capacitance to the network. PFC can sometimes also avoid the need for additional transformer capacity, thereby saving cost. However, capacitance if added more than necessary or too close to the induction motors may damage the induction motors when the power supply is interrupted (de Kock and Strauss, 2004). To prevent connected shunt capacitors from worsening the transients during voltage interruptions, DA can disconnect the capacitors automatically during a severe voltage interruption.

Integrated volt/VAr control (IVVC): Reactive power compensation by shunt capacitors is instrumental in PFC, but at light loading, the same capacitors will increase the voltage to above the allowable limit, causing energy loss. Hence, capacitor banks are usually switched in when the load is heavy, and switched out when otherwise-DA can automate this process as part of IVVC.

In the simplest formulation, IVVC is a minimization problem whose cost function is energy loss, and constraints include voltage limits, maximum number of capacitor switching operations per day, and power factor (Borozan et al., 2001). Krok and Genc's (2011) scheme, which is presumably used in GE's Coordinated Volt VAR Control commercial system (Schneider and Weaver, 2012), consists of multiple optimization stages over a 24-hour schdule period. The first stage is to solve a knapsack problem involving reactive power shortage as the cost function, and capacitor-bank switching schedule as the optimization variable. Reactive power shortage is the difference between target reactive power and the capacitors banks' reactive power output, and the target reactive power is a function of the target power factor and the real power demand forecast. The second stage is to compute the corresponding tap-change schedule of load tap changers and/or voltage regulators, to flatten the average load voltage curve over the schedule period, and maintain the average load voltage at the target level.

V2G ancillary services: EVs can provide frequency regulation and/or voltage control services by participating in the electricity market through an aggregator who has a contract with the grid operator. Based on its objectives that include minimizing system losses, the grid operator dispatches the appropriate regulation signal within the contracted boundary to the aggregator. Based on the regulation signal, the aggregator determines the optimal charging plan.

For frequency regulation, Han et al. (2010) propose a per-EV dynamic programming approach for determining the charging sequence that maximizes revenue. In their model, a charger is turned either off or on at full charging rate. Sortomme and El-Sharkawi (2011) took a different approach, where the aggregator modulates each EV's charging rate around its preferred charging rate (its preferred operating point in ancillary services parlance). This approach maximizes the aggregator's revenue with individual EVs' preferred operating points encoded in the constraints. Targeting energy efficiency specifically in another work (Sortomme et al., 2011), the same authors propose three charging schemes that minimize system losses. It was found that maximizing load factor (linear program) or minimizing load variance (quadratic program) approximately minimizes losses.

Distribution automation (DA): DA is the remote monitoring and control of switched capacitor banks, reclosers, voltage regulators, and other distribution network devices. DA is a part of a larger system called distribution management system, which performs the equivalent functions of an EMS for distribution networks. The goal of DA is to improve the availability, reliability, and efficiency of the distribution system, by addressing the following areas:

- Fault diagnosis and trouble call: It allows the utility to identify and resolve system problems remotely and efficiently.

- Network reconfiguration: It enables load balancing and computer-optimized load shifting to alleviate overload conditions and maintain system stability.

- System restoration: It reconfigures the system after disturbances or interruptions due to failures, accidents or disasters.

- Analysis: It facilitates coordination with DERs, outage management systems, advanced metering infrastructures, demand-side management systems, etc. for data analysis purposes.

The concept of DA first appeared in the 1970s, but DA implementations (e.g., in the U.S. and Australia) only began in the late 1990s (Smallwood and Wennermark, 2010). Until recently, DA implementations have been mainly concerned with the automated control of basic circuit switching functions. EPRI (2004) recently outlined a long-term roadmap to develop technologies for advanced distribution automation (ADA), which is the complete automation of all controllable equipment and functions in a distribution system. The objective of ADA is to enhance service reliability, power quality, and system efficiency, by automating: (i) data preparation in near real time; (ii) optimal decision making; and (iii) the control of distribution operations in coordination with transmission and generation system operations.

EPRI has identified two components critical to ADA: (i) an open communication architecture that enables field 
devices to communicate with substations; and (ii) a redeveloped power system from an electrical architecture standpoint for component interoperability. The focus here is item (i), which covers not only ADA but also substation automation (SA) and V2G. While DA extends beyond distribution substations, SA is chiefly the automation of data acquisition, control, protection, diagnostics and monitoring functions within substations. Nowadays however, $\mathrm{SA}$ is extended to feeder devices beyond substations (Fan et al., 2009). In the next three subsections, communication technologies for DA, SA and V2G are discussed.

\subsection{Communication technologies for DA}

Before committing to any long-term investment in a DA communication infrastructure, a utility has to consider at least the following aspects of the communication technology to avoid the risk of stranded assets: cost, capacity, latency, reliability, robustness and scalability. Security is an important issue, but it can be considered independently of the communication technology.

A distribution network has different characteristics depending on its locale. Typically, urban networks are dense and have a loop/mesh topology, whereas rural networks are sparse and have a radial topology. Reports from several commercial rollouts (Choi et al., 2008; Kim et al., 2009; Smallwood and Wennermark, 2010) recommend fiber-tothe-pole (FTTP) for the loop/mesh topology of urban networks, and wireless communications for rural networks. FTTP networks have the advantages of very high capacity (up to $1600 \mathrm{Gbps}$ ), very low latency, very low bit error ratio $\left(10^{-15}\right)$, and immunity to interference. In comparison, twisted pair cables have lower capacity and higher bit error ratio; telephone lines often delay service restoration due to separate ownership of lines and equipment. Australia's smart grids will use FTTP if the National Broadband Network materializes.

Nevertheless, the high installation cost of FTTP makes power line communication an appealing alternative to utilities, as evidenced by ongoing research initiatives such as the Open PLC European Research Alliance. Power line communication saves cost by reusing existing grid infrastructure. A pilot test of broadband power line communication over medium voltage between a primary and a secondary substation confirms the practicality of the technology for IEC 61850-compliant automation (Giustina et al., 2013). However, the technology faces several challenges (EPRI, 2004; Chao et al., 2013):

- Power lines are inherently noisy and frequencyselective channels. In particular, transformers are lowpass filters that cause severe frequency-dependent attenuation of communication signals.

- Opening of circuit breakers, auto-reclosers, switches etc. ceases communication with de-energized areasthis is known as the "open circuit problem".

- The presence/absence of devices such as capacitor banks causes significant variance of channel conditions, impacting bandwidth and transmission range.

To summarize, Gungor and Lambert (2006) provide a detailed comparison of wireline communication technologies in terms of their suitability for DA.
There is a wealth of options-open or proprietary-in the wireless area. Table 2 compares these options. In Table 2, cost comparison is omitted for the lack of actual figures. One technology that is not listed in Table 2 is satellite communications, because in most situations it is not an attractive option due to its cost, capacity, latency and weather-dependence. However, in extremely remote locations, it might be the only solution. Silver Spring Networks is included in Table 2 because of its large-scale adoption in Australia.

It is clear from reports in the literature so far that there is no single technology that suits all scenarios, making it likely that different technologies are used on different segments of a distribution network. The observed trend is to deploy satellite communications in remote areas without cell-phone coverage; CDMA2000 in remote areas with cellphone coverage; WiMAX or $900 \mathrm{MHz}$ radio in rural areas; and FTTP in urban areas. The final decision of any actual deployment depends on a full cost assessment.

A DA communications architecture is typically two-tier, consisting of an IP-based backbone and field networks. Since many field network protocols expect serial interfaces, a challenge lies in emulating serial communication links across a network, so that neither field network protocols nor application servers need to be modified. The European project REMPLI has developed a "semi-transparent tunnelling" scheme, that is a compromise between pure tunnelling and pure gateway-based protocol translation, to address the problem (Sauter and Lobashov, 2011).

\subsection{Communication technologies for $S A$}

The most important development for SA in recent years is the IEC 61850 series of standards. In the 1990s, as intelligent electronic devices flourished in substations, interoperability between devices from different vendors became a concern. To address this concern, EPRI created Utility Communications Architecture (UCA) version 2.0, but IEC recognized the need for a more general standard and created IEC 61850, based partly on UCA2. A highly accessible introduction to IEC 61850 is available from Mackiewicz (2006).

The standardized architecture consists of a Station Bus and a Process Bus (see Fig. 3). The Station Bus supports communications between protection, control, monitoring, and logging functions, and remote access of these functions; whereas the Process Bus supports collection of voltage, current, and status information via Merging Units, which sample signals at a predefined, synchronized rate. Generic Object-Oriented Substation Events carry status information that can be exchanged peer-to-peer between devices, whereas Sampled Values carry measurements such as voltage, current, and vibration. Three of IEC 61850's most salient features are discussed below.

Abstract communication service interface: The interface allows data objects and services to be defined independent of the underlying communication protocols, but the standard does specify a mapping of abstract data objects and services to ISO 9506, i.e., the Manufacturing Messaging Specification. The specification chooses IEEE 
Table 2. Comparison of wireless communication technologies for DA.

\begin{tabular}{|c|c|c|c|c|c|}
\hline & CDMA2000 & GE-MDS $900 \mathrm{MHz}$ & $\begin{array}{l}\text { Silver Spring } \\
\text { Networks }\end{array}$ & Wi-Fi/IEEE 802.11 & WiMAX/IEEE 802.16 \\
\hline Interoperability & Open standard & Proprietary & Proprietary & Open standard & Open standard \\
\hline Capacity & $\begin{array}{l}76.8 \mathrm{kbps}(80-\mathrm{ms} \\
\text { frame }) \\
153.6 \mathrm{kbps} \\
(40-\mathrm{ms} \text { frame }) \\
307.2 \mathrm{kbps} \\
(20-\mathrm{ms} \text { frame })\end{array}$ & $\begin{array}{l}19.2 \mathrm{kbps}(80 \mathrm{~km}) \\
115 \mathrm{kbps}(48 \mathrm{~km}) \\
1 \mathrm{Mbps}(32 \mathrm{~km})\end{array}$ & $100 \mathrm{kbps}$ & $\begin{array}{l}54 \text { Mbps }(802.11 \mathrm{a}) \\
11 \text { Mbps }(802.11 \mathrm{~b}) \\
54 \text { Mbps }(802.11 \mathrm{~g}) \\
72 \text { Mbps }(802.11 \mathrm{n})\end{array}$ & 9 Mbps \\
\hline Latency & $\begin{array}{l}\text { Hundreds of } \\
\text { milliseconds }\end{array}$ & Tens of milliseconds & $\begin{array}{l}\text { Tens of } \\
\text { milliseconds }\end{array}$ & Milliseconds & Milliseconds \\
\hline $\begin{array}{l}\text { Interference re- } \\
\text { jection }\end{array}$ & $\begin{array}{l}\text { DSSS, } 2 \mathrm{GHz} \\
\text { frequency band } \\
\text { allows frequency } \\
\text { band re-use }\end{array}$ & FHSS, 902-928 MHz & $\begin{array}{l}\text { FHSS, } \\
902-928 \mathrm{MHz}\end{array}$ & $\begin{array}{l}\text { 802.11a: ODFM, } 5 \mathrm{GHz} \\
\text { 802.11b: DSSS, } 2.4 \mathrm{GHz} \\
\text { 802.11g: OFDM/DSSS, } 2.4 \mathrm{GHz} \\
\text { 802.11n: OFDM, } 2.4 / 5 \mathrm{GHz} \\
\text { *2.4 GHz band is crowded; } 5 \\
\mathrm{GHz} \text { less so }\end{array}$ & OFDM, 3.65-3.70 GHz \\
\hline $\begin{array}{l}\text { Transmission } \\
\text { range }\end{array}$ & $\begin{array}{l}\text { Nation-wide } \\
\text { service coverage }\end{array}$ & $80 \mathrm{~km}$ & Unknown & $\begin{array}{l}\text { 802.11a: } 120 \mathrm{~m} \\
802.11 \mathrm{~b} / \mathrm{g}: 140 \mathrm{~m} \\
802.11 \mathrm{n}: 250 \mathrm{~m}\end{array}$ & $20 \mathrm{~km}$ \\
\hline Configuration & $\begin{array}{l}\text { Point-to- } \\
\text { multipoint }\end{array}$ & $\begin{array}{l}\text { Point-to-point, } \\
\text { point-to-multipoint }\end{array}$ & Point-to-point & $\begin{array}{l}\text { Point-to-point, } \\
\text { point-to-multipoint }\end{array}$ & Point-to-multipoint \\
\hline $\begin{array}{l}\text { Supporting } \\
\text { case }\end{array}$ & Choi et al. (2008) & $\begin{array}{l}\text { Smallwood and } \\
\text { Wennermark (2010) }\end{array}$ & Unknown & Unknown & \begin{tabular}{|lll}
$\begin{array}{l}\text { Clark } \\
(2010)\end{array}$ & and & Pavlovski \\
\end{tabular} \\
\hline
\end{tabular}

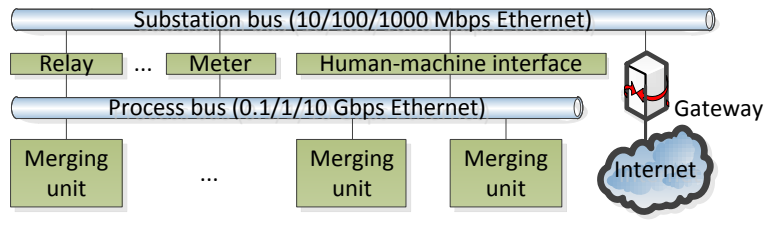

Fig. 3. IEC 61850 architecture of a substation.

802.3 (Ethernet) for the physical and data link layers, and TCP/IP for the network and transport layers.

Object-oriented data modelling: Each IEC 61850 device is modelled using a Physical Device object. Each physical device contains one or more Logical Device objects. Each logical device contains one or more Logical Nodes, and each logical node contains one or more Data elements. Naming a Data element in the order of its parent Physical Device, its parent Logical Device, its parent Logical Node, and its own Data name, is user-friendly and allows every Data element to be mapped to a uniquely named Manufacturing Messaging Specification variable object.

Substation automation system configuration: The XML-based Substation Configuration Language is used to specify/configure (i) substation devices in terms of capabilities and communications, (ii) functionalities as represented by Logical Nodes, and (iii) substation topology.

IEC 61850 does not specify the representation of control and automation logic; this gap is filled by IEC 61499, a software architecture standard for industrial control systems. The architecture is based on Function Blocks, which are self-contained software modules, each of which acts as a finite state machine providing an input interface and an output interface. Function Blocks provide a natural mapping to Logical Nodes.
4.3 Communication technologies for V2G

Communication requirements between the system operator and an aggregator are sufficiently served by the Internet. Between an aggregator and EVs, three pathways are involved:

- between an EV and a charging station, Bluetooth is suitable due to its ubiquity in EVs;

- between charging stations; and

- between a charging station and the aggregator's energy management system, there are many options.

For example, the smart charging infrastructure called WINSmartEV ${ }^{\mathrm{TM}}$ at the Smart Grid Energy Research Center, UCLA, is interoperable with a variety of communication technologies such as WiFi, 3G, 4G, ZigBee, and power line communication. WINSmartEV ${ }^{\mathrm{TM}}$ provides two types of bidirectional connectivity: (i) between any pair of charging stations; and (ii) between the charging stations and a cloud-based energy management system that hosts and executes smart charging algorithms (Chung et al., 2013). EV owners can interact with the aggregator via an "Energy Service Interface" (Lee et al., 2013).

As EVs can come with non-interoperable communication technologies, it is a challenge to ensure that devices running within a single network can communicate with each other and function as a single system. ZigBee and HomePlug alliances are working towards interoperability protocols. Furthermore, when the network size grows, data forwarding problems arise, causing packet loss. This is commonly observed with heavy traffic in dense mesh networks, so a data forwarding scheme should be selected carefully (Kim et al., 2012). Another variable that impacts performance of low-power networks in the power grid is scalability. A Delaunay Triangulation network has the desirable property where the complexity of a group join or group leave operation is constant (Kim et al., 2012). 


\section{RESEARCH CHALLENGES AND OPPORTUNITIES}

Based on the state of the art presented so far, we arrive at the following research challenges and opportunities pertaining to energy-efficient smart grids.

Generation: IEEE Std 1547-2003 specifies the requirements for DER-grid connections, but falls short of addressing several key interconnection issues, such as potential overvoltages, and interconnection transformer choices (Mozina, 2010). There is extensive research and on-going standardization effort in this area. There is keen interest in applying multi-agent systems, with the support of broadband communication networks, to voltage control, as well as active and reactive power control (Nguyen et al., 2008). Self-reconfigurable microgrids, DC-based microgrids, coping with the inherent uncertainties and diverse characteristics of a large number of microsources, are important topics (Zamora and Srivastava, 2010).

Transmission: WAMS deployments are being reported regularly. At the same time, more applications for realtime, wide-area monitoring, control and protection are being developed. However, achieving real-time communication for these applications remains a challenge, especially when shared networks are used. The challenge is even greater when phasor data have to be shared across international borders. Researchers of the North American SynchroPhasor Initiative (NASPI), whose mission is to create a robust, widely available and secure synchronized data infrastructure, face the challenge of meeting the stringent network performance requirements, as well as addressing the network management and cyber security issues. Transmission line monitoring is an excellent application of sensor networks, but difficult access to transmission lines for experimentation means research in this area is not open to most sensor network research groups.

Distribution: The viability of V2G technology depends on a working ancillary services market, where market regulations applied to aggregators allow maximum aggregator profits to coexist with maximum consumer and utility benefits. Much work in DA is being devoted to (i) pilot-testing implementations conforming to IEC 61850 and IEEE Std 1646 (delivery time performance requirements), e.g., the FREEDM testbed (Wang et al., 2011); and (ii) development issues surrounding the standard, e.g., the implementation of automatic control functions specified using petri nets in the IEC 61850 environment (de Sá and and Cartaxo, 2011). There is ongoing research on constructing a formal semantic model for IEC 61499 so that applications written using the standard can be formally verified (Vyatkin, 2009). More research questions are expected to be carved out as the industry gains more implementation experience.

\section{CONCLUSION}

This review bears witness to the trend of increasing convergence between sensing, control and communication technologies in the power grid, but a successful integration of these technologies requires a holistic understanding of various smart grid subsystems. The review is written with the goal of facilitating such an understanding, by discussing control and communication techniques used for generation, transmission and distribution.

On the generation side, distribution generation and microgrid techniques increase penetration of distributed energy resources, allowing scarce fossil fuels to be used efficiently. On the transmission side, wide-area monitoring and control techniques enhance operators' situational awareness, facilitate optimal system planning, energy-efficient electrical transmission, as well as stabilization of oscillation, voltage and frequency. Distribution automation and substation automation techniques facilitate fault diagnosis, network reconfiguration, system restoration and analysis, reducing energy loss in distribution networks.

\section{REFERENCES}

Acha, S., Green, T., and Shah, N. (2010). Effects of optimised plug-in hybrid vehicle charging strategies on electric distribution network losses. In 2010 IEEE PES Transmission and Distribution Conference and Exposition, 1-6. doi:10.1109/TDC.2010.5484397.

Ackermann, T., Andersson, G., and Söder, L. (2001). Distributed generation: a definition. Electric Power Systems Research, 57, 195-204.

Andersson, G., Ilić and, M.D., Madani, V., and Novosel, D. (2011). Network systems engineering for meeting the energy and environmental dream [scanning the issue]. Proc. IEEE, 99(1), 7-14.

Ayres, H.M., Freitas, W., Almeida, M.C.D., and Silva, L.C.P.D. (2010). Method for determining the maximum allowable penetration level of distributed generation without steady-state voltage violations. IET Generation, Transmission \& Distribution, 4(4), 495-508.

Birman, K., Chen, J., Hopkinson, E., Thomas, R., Thorp, J., Van Renesse, R., and Vogels, W. (2005). Overcoming communications challenges in software for monitoring and controlling power systems. Proc. IEEE, 93(5), 10281041.

Borozan, V., Baran, M., and Novosel, D. (2001). Integrated volt/VAr control in distribution systems. In 2001 IEEE Power Engineering Society Winter Meeting, volume 3, 1485-1490.

Budischak, C., Sewell, D., Thomson, H., Mach, L., Veron, D.E., and Kempton, W. (2013). Cost-minimized combinations of wind power, solar power and electrochemical storage, powering the grid up to $99.9 \%$ of the time. Journal of Power Sources, 225(0), 60 - 74.

Chao, C.W., Ho, Q.D., and Le-Ngoc, T. (2013). Challenges of power line communications for advanced distribution automation in smart grid. In 2013 IEEE Power and Energy Society General Meeting (PES), 1-5.

Choi, T.I., Lee, K.Y., Lee, D.R., and Ahn, J.K. (2008). Communication System for Distribution Automation Using CDMA. IEEE Trans. Power Del., 23(2), 650656.

Chung, C.Y., Chu, P., and Gadh, R. (2013). Design of smart charging infrastructure hardware and firmware design of the various current multiplexing charging system. In Seventh Global Conference on Power Control and Optimization (PCO 2013).

Chung, I.Y., Liu, W., Cartes, D., Collins, E.G., J., and Moon, S.I. (2010). Control methods of inverterinterfaced distributed generators in a microgrid system. IEEE Trans. Ind. Appl., 46(3), 1078-1088. 
Clark, A. and Pavlovski, C. (2010). Wireless networks for the smart energy grid: Application aware networks. In Proceedings of the International MultiConference of Engineers and Computer Scientists (IMECS 2010), volume II. Newswood Limited.

de Kock, J. and Strauss, C. (2004). Practical Power Distribution for Industry. Elsevier.

De La Ree, J., Centeno, V., Thorp, J., and Phadke, A. (2010). Synchronized phasor measurement applications in power systems. IEEE Transactions on Smart Grid, 1(1), 20-27. doi:10.1109/TSG.2010.2044815.

de Sá and, J. and Cartaxo, R. (2011). Implementing Substations Automatic Control Functions Designed With Petri Nets on IEC 61850. IEEE Trans. Power Del. 26(2), 1119-1127. doi:10.1109/TPWRD.2010.2090952.

Dorf, R.C. and Bishop, R.H. (2008). Modern Control Systems. Pearson Education International, 11th edition.

Elizondo, D., Gentile, T., Candia, H., and Bell, G. (2010). Overview of robotic applications for energized transmission line work - technologies, field projects and future developments. In 1st International Conference on Applied Robotics for the Power Industry (CARPI), 1-7.

EPRI (2004). Technical and system requirements for advanced distribution automation. Final Report.

EPRI (2009). Sensor technologies for a smart transmission system. white paper.

Fan, J., du Toit, W., and Backscheider, P. (2009). Distribution substation automation in smart grid. The Protection $\&$ Control Journal.

Farhangi, H. (2010). The path of the smart grid. IEEE Power and Energy Magazine, 8(1), 18-28.

Gao, J., Xiao, Y., Liu, J., Liang, W., and Chen, C.P. (2012). A survey of communication/networking in Smart Grids. Future Gener. Comput. Syst., 28(2), 391404. doi:10.1016/j.future.2011.04.014.

Giustina, D., Ferrari, P., Flammini, A., Rinaldi, S., and Sisinni, E. (2013). Automation of distribution grids with IEC 61850: A first approach using broadband power line communication. IEEE Trans. Instrum. Meas., 62(9), 2372-2383.

Gungor, V. and Lambert, F. (2006). A survey on communication networks for electric system automation. Computer Networks, 50(7), 877-897.

Han, S., Han, S., and Sezaki, K. (2010). Development of an optimal vehicle-to-grid aggregator for frequency regulation. IEEE Transactions on Smart Grid, 1(1), 65-72. doi:10.1109/TSG.2010.2045163.

Hauer, J., Demeure, C., and Scharf, L. (1990). Initial results in prony analysis of power system response signals. IEEE Trans. Power Syst., 5(1), 80-89. doi: $10.1109 / 59.49090$.

Hauser, C., Bakken, D., and Bose, A. (2005). A failure to communicate: next generation communication requirements, technologies, and architecture for the electric power grid. IEEE Power Energy Mag., 3(2), 47-55.

He, J. and Li, Y.W. (2012). An enhanced microgrid load demand sharing strategy. IEEE Trans. Power Electron., 27(9), 3984-3995.

He, M., Reutzel, E., Jiang, X., Katz, R., Sanders, S., Culler, D., and Lutz, K. (2008). An architecture for local energy generation, distribution, and sharing. In IEEE Energy 2030 Conference, 1-6.
Huang, Y., Xu, Z., and Pan, W. (2005). A practical analysis method of low frequency oscillation for large power systems. In Power Engineering Society General Meeting, 2005. IEEE, 1623-1629 Vol. 2.

Hung, K., Lee, W., Li, V., Lui, K., Pong, P., Wong, K., Yang, G., and Zhong, J. (2010). On wireless sensors communication for overhead transmission line monitoring in power delivery systems. In 2010 First IEEE International Conference on Smart Grid Communications (SmartGridComm), 309-314.

Ipakchi, A. and Albuyeh, F. (2009). Grid of the future. IEEE Power and Energy Magazine, 7(2), 52-62.

Karlsson, D., Hemmingsson, M., and Lindahl, S. (2004). Wide area system monitoring and control - terminology, phenomena, and solution implementation strategies. IEEE Power and Energy Magazine, 2(5), 68-76.

Kawamura, A., Haneyoshi, T., and Hoft, R. (1988). Deadbeat controlled PWM inverter with parameter estimation using only voltage sensor. IEEE Trans. Power Electron., 3(2), 118 -125. doi:10.1109/63.4341.

Kim, M., Metzner, J., and Lee, K. (2009). Design and implementation of a last-mile optical network for distribution automation. IEEE Trans. Power Del., 24(3), 1198-1205. doi:10.1109/TPWRD.2008.2008487.

Kim, Y.J., Lee, J., Atkinson, G., Kim, H., and Thottan, M. (2012). SeDAX: a scalable, resilient, and secure platform for Smart grid communications. IEEE J. Sel. Areas Commun., 30(6), 1119-1136.

Korba, P. (2007). Real-time monitoring of electromechanical oscillations in power systems: first findings. Generation, Transmission Distribution, IET, 1(1), 8088. doi:10.1049/iet-gtd:20050243.

Krok, M.J. and Genc, S. (2011). A coordinated optimization approach to Volt/VAr control for large power distribution networks. In American Control Conference (ACC 2011), 1145-1150.

Larsson, M., Rehtanz, C., and Bertsch, J. (2002). Realtime voltage stability assessment of transmission corridors. In IFAC Symp. Power Plants and Power Systems Control.

Lasseter, R. (2002). Microgrids. In IEEE Power Engineering Society Winter Meeting, volume 1, 305-308.

Law, Y.W., Alpcan, T., Lee, V.C., Lo, A., Marusic, S., and Palaniswami, M. (2012). Demand response architectures and load management algorithms for energy-efficient power grids: A survey. In 2012 Seventh International Conference on Knowledge, Information and Creativity Support Systems (KICSS), 134-141.

Lee, E.K., Gadh, R., and Gerla, M. (2013). Energy service interface: Accessing to customer energy resources for smart grid interoperation. IEEE J. Sel. Areas Commun., 31(7), 1195-1204.

Lee, S.C., Kim, S., and Kim, S. (2011). Demand side management with air conditioner loads based on the queuing system model. Power Systems, IEEE Transactions on, 26(2), 661-668. doi:10.1109/TPWRS.2010.2066583.

Lee, S.H. and Park, J.W. (2009). Selection of optimal location and size of multiple distributed generations by using kalman filter algorithm. IEEE Trans. Power Syst., 24(3), 1393-1400.

Leon, R., Vittal, V., and Manimaran, G. (2007). Application of sensor network for secure electric energy infrastructure. IEEE Trans. Power Del., 22(2), 1021- 
1028. doi:10.1109/TPWRD.2006.886797.

Li, W.H., Tajbakhsh, A., Rathbone, C., and Vashishtha, Y. (2010). Image processing to automate condition assessment of overhead line components. In 1st International Conference on Applied Robotics for the Power Industry (CARPI), 1-6.

Mackiewicz, R. (2006). Overview of IEC 61850 and Benefits. In IEEE PES Power Systems Conference and Exposition (PSCE '06), 623-630.

Martinez, C., Parashar, M., Dyer, J., and Coroas, J. (2005). Phasor Data Requirements for Real Time WideArea Monitoring, Control and Protection Applications. White paper, EIPP - Real Time Task Team.

Marwali, M., Jung, J.W., and Keyhani, A. (2004). Control of distributed generation systems - part ii: Load sharing control. IEEE Trans. Power Electron., 19(6), 15511561.

Massoud Amin, S. and Wollenberg, B. (2005). Toward a smart grid: power delivery for the 21st century. IEEE Power and Energy Magazine, 3(5), 34-41.

Mozina, C. (2010). Impact of green power distributed generation. IEEE Ind. Appl. Mag., 16(4), 55-62.

Nguyen, P., Myrzik, J., and Kling, W. (2008). Coordination of voltage regulation in Active Networks. In IEEE/PES Transmission and Distribution Conference and Exposition, 1-6. doi:10.1109/TDC.2008.4517041.

Peng, J.C.H. and Nair, N. (2009a). Comparative assessment of kalman filter and prony methods for power system oscillation monitoring. In Power Energy Society General Meeting, 2009. PES '09. IEEE, 1-8. doi: 10.1109/PES.2009.5275656.

Peng, J.H. and Nair, N.K. (2009b). Adaptive sampling scheme for monitoring oscillations using prony analysis. Generation, Transmission Distribution, IET, 3(12), 1052-1060. doi:10.1049/iet-gtd.2009.0174.

Pota, H.R. (2013). Droop control for islanded microgrids. In IEEE Power Engineering Society General Meeting, 1-5. Vancouver, Canada.

Quezada, V.H.M., Abbad, J.R., and Román, T.G.S. (2006). Assessment of energy distribution losses for increasing penetration of distributed generation. IEEE Trans. Power Syst., 21(2), 533-540.

Roy, N.K., Pota, H.R., Mahmud, M.A., and Hossain, M.J. (2013a). Key factors affecting voltage oscillations of distribution networks with distributed generation and induction motor loads. International Journal of Electrical Power \&6 Energy Systems, 53, 515-528.

Roy, N.K., Pota, H.R., Mahmud, M.A., and Hossain, M.J. (2013b). Voltage control of emerging distribution systems with induction motor loads using robust LQG approach. International Transactions on Electrical Energy Systems.

Saber, A.Y. and Venayagamoorthy, G.K. (2010). Intelligent unit commitment with vehicle-to-grid -a costemission optimization. Journal of Power Sources, 195(3), 898-911. doi:10.1016/j.jpowsour.2009.08.035.

Santoso, S., Beaty, H.W., Dugan, R.C., and McGranaghan, M.F. (2002). Electrical Power Systems Quality. McGraw-Hill, 2nd edition.

Sauter, T. and Lobashov, M. (2011). End-to-end communication architecture for smart grids. IEEE Trans. Ind. Electron., 58(4), 1218-1228. doi: 10.1109/TIE.2010.2070771.
Schmutzler, J., Wietfeld, C., and Andersen, C. (2012). Distributed energy resource management for electric vehicles using IEC 61850 and ISO/IEC 15118. In 2012 IEEE Vehicle Power and Propulsion Conference (VPPC), 1457-1462.

Schneider, K. and Weaver, T. (2012). Volt-VAR optimization on American Electric Power feeders in Northeast Columbus. In 2012 IEEE PES Transmission and Distribution Conference and Exposition (TED), 1-8.

Sickinger, T. (2010). Too much of a good thing: Growth in wind power makes life difficult for grid managers. The Oregonian. URL goo.gl/LwQSy.

Smallwood, C. and Wennermark, J. (2010). Benefits of distribution automation. IEEE Industry Applications Magazine, 16(1), 65-73. doi:10.1109/MIAS.2009.934970.

Sortomme, E. and El-Sharkawi, M. (2011). Optimal charging strategies for unidirectional vehicle-to-grid. IEEE Transactions on Smart Grid, 2(1), 131-138.

Sortomme, E., Hindi, M., MacPherson, S., and Venkata, S. (2011). Coordinated charging of plug-in hybrid electric vehicles to minimize distribution system losses. IEEE Transactions on Smart Grid, 2(1), 198-205.

Tan, S.K., Sooriyabandara, M., and Fan, Z. (2011). M2M Communications in the Smart Grid: Applications, Standards, Enabling Technologies, and Research Challenges. International Journal of Digital Multimedia Broadcasting, 2011. Article ID 289015, 8 pages.

Timbus, A., Liserre, M., Teodorescu, R., Rodriguez, P., and Blaabjerg, F. (2009). Evaluation of current controllers for distributed power generation systems. IEEE Trans. Power Electron., 24(3), 654-664.

Tomić, J. and Kempton, W. (2007). Using fleets of electric-drive vehicles for grid support. Journal of Power Sources, 168(2), 459-468.

U.S. Department of Energy (2008). The Smart Grid: An Introduction.

Venayagamoorthy, G. (2011). Dynamic, stochastic, computational, and scalable technologies for smart grids. IEEE Comput. Intell. Mag., 6(3), 22-35. doi: 10.1109/MCI.2011.941588.

Vovos, P., Kiprakis, A., Wallace, A., and Harrison, G. (2007). Centralized and distributed voltage control: Impact on distributed generation penetration. IEEE Trans. Power Syst., 22(1), 476-483.

Vyatkin, V. (2009). The IEC 61499 standard and its semantics. IEEE Trans. Ind. Electron., 3(4), 40-48.

Wang, W., Xu, Y., and Khanna, M. (2011). A survey on the communication architectures in smart grid. Computer Networks, 55(15), 3604-3629.

Zamora, R. and Srivastava, A.K. (2010). Controls for microgrids with storage: Review, challenges, and research needs. Renewable and Sustainable Energy Reviews, 14(7), 2009-2018.

Zhang, B. and Baillieul, J. (2013). A novel packet switching framework with binary information in demand side management. In 52nd IEEE Conference on Decision and Control. To appear.

Zima, M., Larsson, M., Korba, P., Rehtanz, C., and Andersson, G. (2005). Design aspects for wide-area monitoring and control systems. Proc. IEEE, 93(5), 980-996. doi:10.1109/JPROC.2005.846336. 